\title{
Value of magnetic resonance imaging indices of left renal vein entrapment in the diagnosis of nutcracker syndrome in children
}

\author{
Ren Wang ${ }^{1 \#}$, Meiqiu Wang ${ }^{2 \#}$, Zhengkun Xia ${ }^{3}$, Chunlin Gao ${ }^{3}$, Qianhuining Kuang ${ }^{1}$, Xiang Fang ${ }^{3}$, Min Yu $^{1}$, \\ Yinchao Peng ${ }^{2}$, Xiaojie $\mathrm{Li}^{4}$, Yaqin $\mathrm{Wei}^{3}$, Tao Ju${ }^{1}$ \\ ${ }^{1}$ Department of Pediatrics, Jinling Hospital, Nanjing Medical University, Nanjing, China; ${ }^{2}$ Department of Pediatrics, Jinling Hospital, The First \\ School of Clinical Medicine, Southern Medical University, Nanjing, China; ${ }^{3}$ Department of Pediatrics, Jinling Hospital, Nanjing University, School \\ Medical, Nanjing, China; ${ }^{4}$ Department of Pediatrics, Affiliated Jinling Hospital, Medical School of Nanjing University, China \\ Contributions: (I) Conception and design: R Wang; (II) Administrative support: Z Xia, C Gao; (III) Provision of study materials or patients: M Wang, \\ Q Kuang, X Fang, M Yu; (IV) Collection and assembly of data: Y Peng, X Li, Y Wei, T Ju; (V) Data analysis and interpretation: R Wang, M Wang; (VI) \\ Manuscript writing: All authors; (VII) Final approval of manuscript: All authors. \\ \#These authors contributed equally to this work. \\ Correspondence to: Dr. Zhengkun Xia or Dr. Chunlin Gao. Nanjing Jinling Hospital, 305 Zhongshan East Road, Xuanwu District, Nanjing, China. \\ Email: njxzk@126.com; shuangmu34@163.com.
}

Background: Nutcracker syndrome (NCS) is also known as left renal vein (LRV) compression syndrome. Magnetic resonance imaging (MRI) can better depict the anatomical structure of soft tissues in the area of compression. Diagnosis of NCS using MRI in adults is not uncommon. However, there are few studies on the diagnosis of NCS using MRI in children. Therefore, we conducted this study to evaluate the clinical value of the MRI indices of the LRV in the diagnosis of NCS in children.

Methods: This study was a single-center retrospective analysis. One hundred seventy-four patients with suspected NCS were enrolled from January 2017 to July 2020. The inclusion criteria for suspected NCS were mainly based on clinical symptoms or signs, laboratory examinations and imaging reports. Other diseases that may cause hematuria and/or proteinuria were excluded. We grouped the patients based on the diagnostic criteria for NCS into a nutcracker group and a control group. The receiver operating characteristic (ROC) curve was constructed for evaluating the value of MRI indices in the diagnosis of NCS.

Results: The majority of NCS patients presented with orthostatic proteinuria (OP) (67.2\%), followed by hematuria (55.2\%), abdominal pain (19.0\%), and left flank pain (15.5\%). The areas under the curve (AUCs) for the superior mesenteric artery (SMA) angle, beak sign, and compression ratio (CR) in the diagnosis of NCS were $0.870,0.895$, and 0.878 , respectively, and the best cutoff values of the SMA angle and CR were 36.8 and 3.99, respectively. The specificities of SMA angle $<36.8^{\circ}$, beak sign, CR $>3.99$, SMA angle $<36.8^{\circ}$ and beak sign, SMA angle $<36.8^{\circ}$ and CR $>3.99$, and beak sign and CR $>3.99$ were $82.5 \%, 93.8 \%, 93.8 \%$, $97.9 \%, 95.9 \%$ and $97.9 \%$, respectively.

Conclusions: Children with SMA angles less than $36.8^{\circ}$, beak signs and CR greater than 3.99 should be highly suspected of having NCS. Among these parameters, "beak sign" showed the highest diagnostic accuracy by MRI, and the combination of any two of the above parameters achieved a higher specificity than the single parameters.

Keywords: Nutcracker syndrome (NCS); left renal vein (LRV); magnetic resonance imaging (MRI); children

Submitted Dec 18, 2020. Accepted for publication Apr 09, 2021.

doi: $10.21037 /$ tp-20-466

View this article at: http://dx.doi.org/10.21037/tp-20-466 


\section{Introduction}

Nutcracker syndrome (NCS), also known as the left renal vein (LRV) entrapment syndrome, refers to the compression of the LRV and its associated characteristic symptoms (1). This condition leads to increased venous pressure in the LRV due to compression of the LRV by the superior mesenteric artery (SMA) and abdominal aorta (anterior NCS) or by the vertebral column and aorta (posterior NCS). It is hypothesized that LRV hypertension may result in hematuria, proteinuria, left flank pain, abdominal pain, and other symptoms associated with the nutcracker anatomy (2). The true prevalence of NCS remains unknown, and previous studies have reported that NCS is a rare condition (3). However, an increasing number of patients are being diagnosed after treatment failure for nephropathies $(4,5)$. Therefore, it is likely that the exact prevalence of NCS may be higher than previously thought. NCS can occur at any age, with prevalence peaking in the second and third decades of life (3). In several previous studies, the proportion of female patients was higher. However, one study showed that there was no sex difference in the occurrence of NCS (6).

The diagnosis of NCS requires a combination of clinical history, laboratory data, and imaging reports, of which the imaging technique plays a crucial role. Compression of the LRV can be determined by doppler ultrasonography (DUS), computed tomography (CT), magnetic resonance imaging (MRI), phlebography, and intravascular ultrasound. However, there are no definitive imaging diagnostic criteria for NCS. DUS is recommended as the first diagnostic tool for NCS, with a sensitivity of $69-90 \%$ and a specificity of $89-100 \%$, but its index and range of values are highly variable (7). Although phlebography and intravascular ultrasound are the gold standards in the diagnosis of NCS, they are not commonly used in clinical practice because they are invasive. Both CT and MRI can portray the compression of the LRV by axial imaging. The SMA angle, compression ratio (CR), and beak sign are the specific findings of axial imaging, and several studies have reported that these techniques can diagnose LRV compression with high accuracy $(1,2)$. However, CT can lead to radiation exposure, and there is a risk of allergy to the contrast agent in children.

MRI is a radiation-free and multiplane imaging technique that can better depict the anatomical structure of the soft tissues in the area of compression. Significant results produced by the MRI were very similar to those produced by $3 \mathrm{D}-\mathrm{CT}$, with similar accuracy $(1,2)$. The diagnosis of NCS using MRI is not uncommon in adults. However, there are few studies on the diagnosis of NCS using MRI in children. Therefore, we retrospectively analyzed the diagnostic value of LRV MRI indices in children with NCS to provide more objective radiological criteria for the diagnosis. We present the retrospective case study in accordance with the STARD reporting checklist (available at http://dx.doi.org/10.21037/tp-20-466).

\section{Methods}

\section{Participants}

This single-center retrospective study recruited all patients admitted to the Department of Pediatrics at Nanjing Jinling Hospital from January 2017 to July 2020. Patients with suspected NCS were enrolled in the cohort. The study was conducted in accordance with the Declaration of Helsinki (as revised in 2013). The study was approved by the Institutional Ethics Committee of Nanjing Medical University (2020NZGKJ-004) and individual consent for this retrospective analysis was waived.

The diagnosis of suspected NCS was made by the attending physician at the time of initial diagnosis based on clinical symptoms or signs (macroscopic hematuria, flank pain, abdominal pain, pelvic pain, etc.), laboratory examinations (microscopic hematuria and proteinuria), and imaging findings (if DUS was performed, the compression of the LRV was observed). Exclusion of primary or secondary kidney diseases, infections, presence of toxins, urolithiasis, malignancies, and other diseases that might lead to hematuria and/or proteinuria was done. Patients with concurrent proteinuria and hematuria and a positive family history of renal disease were also excluded. The former were excluded because several studies have reported that this phenomenon is most likely due to renal disease. All enrolled patients underwent LRV MRI examination at the time of admission. Patients with incomplete clinical data and younger children who did not cooperate with the MRI examination were excluded.

\section{Data collection}

Demographic characteristics, main clinical symptoms and signs at the time of admission, laboratory examinations, and radiologic findings of all enrolled patients were collected. General characteristics included sex, age of onset, and body 
mass index (BMI). Clinical symptoms associated with the nutcracker anatomy were recorded, including orthostatic proteinuria (OP), left flank pain, and abdominal pain. The laboratory data included hematuria, proteinuria, and 24-h urinary protein. The radiologic findings of MRI, including the angle between the abdominal aorta and the SMA, the diameter of the hilar and aortomesenteric (AM) portion of the LRV, and the "beak sign", were assessed. All data were collected at the time closest to the imaging procedure.

\section{Study design}

Patients diagnosed with NCS were enrolled in the nutcracker group. The diagnostic criteria for NCS are detailed below. (I) Radiological examination confirmed the LRV entrapment. The imaging results of MRI were as follows: (i) LRV diameter ratio (hilar to aortomesenteric ratio) $>4.0$ (8), (ii) angle between the abdominal aorta and the SMA less than $35^{\circ}$ (2), and (iii) the presence of "beak sign" (8). The patients who satisfied 2 or more of the above criteria were diagnosed with LRV entrapment. (II) The following were considered characteristic symptoms or signs: hematuria and/or OP, presence or absence of flank pain, abdominal pain, pelvic pain, and gonadal varices. Two experienced radiologists measured the LRV diameter ratio and the SMA angle, and the average values were used. When there was a disagreement on a parameter (e.g., beak sign) by the two consultants the decision was made by a more experienced radiologist. NCS was diagnosed by two experienced nephrologists. Similarly, if the two people disagreed on a parameter, a decision was made as described above. The patients who were not diagnosed with NCS were included in the control group.

The clinical characteristics, laboratory examinations, and imaging characteristics obtained by MRI in the nutcracker group and the control group were compared. The receiver operating characteristic (ROC) curve was constructed to determine the values of the MRI indices (SMA angle, beak sign, and CR) in the diagnosis of NCS.

\section{Relevant definitions}

OP was defined as normal protein excretion in the supine posture but increased protein excretion after the patient was in the upright position for at least four to six hours $(9,10)$. The persistent presence of more than 3 red cells per high power field (HPF) in centrifuged urine was defined as hematuria. Concurrent proteinuria and hematuria were defined as qualitatively positive protein $(\geq 1+)$ and red cells $>3 / \mathrm{HPF}$ in the morning urine. The "beak sign" refers to the triangular sign formed by the sudden narrowing of the LRV at the angle between the abdominal aorta and the SMA.

\section{Image analysis}

Renal MRI examinations were performed for all patients using a GE Discovery MR750 3.0 t magnetic resonance apparatus and 32-channel body coils. T2-weighted imaging (T2WI) is a routine sequence used to observe beak signs and measure the diameters of the compressed and hilar parts of the LRV. Inflow inversion recovery (IFIR) was used to measure the angle between the abdominal aorta and the SMA. Two values were calculated (11): (I) the CR was defined as the ratio of the diameter of the hilar portion to the diameter of the AM portion, representing the degree of the LRV compression and (II) the degree of compression was calculated as the change in compression (diameter at the hilar portion-diameter at the AM portion) divided by the diameter of the hilar portion.

$\mathrm{CR}=$ diameter at the hilar portion/diameter at the AM portion

Degree of compression $=($ diameter at the hilar portion diameter at the AM portion)/diameter at the hilar portion

\section{Statistical analysis}

SPSS 19.0 software was used for the statistical analysis. Normally distributed variables are presented as the mean \pm $\mathrm{SD}$ and were compared using Student's $t$-test. Continuous variables with skewed distributions are expressed as medians and ranges, and the nonparametric test was used for the comparison. Categorical variables are presented as percentages and were compared using the chi-square test. The ROC curve was used to assess the value of MRI indices in the diagnosis of NCS. The optimal cutoff value is the point closest to the upper left corner of the ROC curve, where sensitivity and specificity are high and false positives and false negatives are minimal to obtain the cutoff value of the SMA angle and CR. All P values were two-sided and values $<0.05$ were considered statistically significant.

\section{Results}

The flow diagram for the inclusion of participants is shown in Figure 1. A total of 4,431 patients were admitted to the department from January 2017 to July 2020. Based on the 


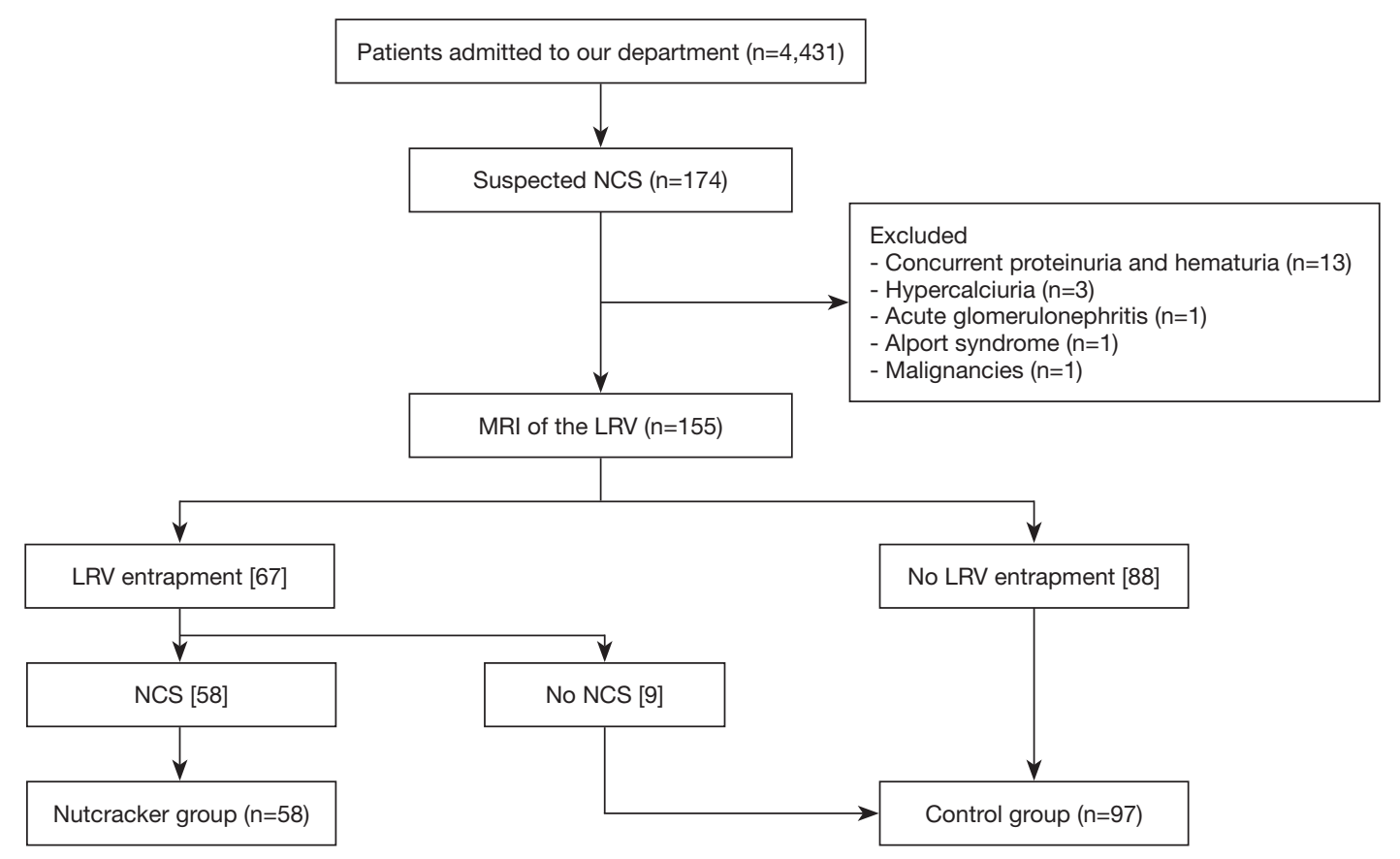

Figure 1 Flow diagram for inclusion of participants. NCS, nutcracker syndrome; MRI, magnetic resonance imaging; LRV, left renal vein.

inclusion criteria for suspected NCS, a total of 174 patients were suspected of having NCS. However, 13 of them were excluded for concurrent proteinuria and hematuria, 3 were excluded for hypercalciuria, 1 was excluded for acute glomerulonephritis, 1 was excluded for Alport syndrome, and 1 was excluded for malignancy. Subsequently, 155 patients were included in the analysis. MRI of the 155 patients showed that 67 patients had LRV compression, and 88 patients had no LRV compression. Of the patients with LRV compression, 58 patients met the diagnostic criteria for NCS, and 9 patients were not diagnosed with NCS. Patients diagnosed with NCS were included in the nutcracker group $(\mathrm{n}=58)$, while those with uncompressed LRV and those with compressed LRV but clinically incompatible with the NCS diagnosis were included in the control group $(\mathrm{n}=97)$.

\section{Baseline characteristics and clinical symptoms}

The baseline characteristics of the two groups of patients are listed in Table 1. There were no differences in sex or age between patients in the nutcracker and control groups. The BMI of NCS patients was lower than that of control patients $(\mathrm{P}=0.020)$. A majority of NCS patients had OP $(67.2 \%)$, followed by hematuria $(55.2 \%)$, abdominal pain
(19.0\%), and left flank pain (15.5\%). The frequency of the above symptoms was significantly lowered in the control group.

\section{Imaging characteristics}

The imaging characteristics of the two groups of patients are listed in Table 2. In the nutcracker group, the mean angle between the abdominal aorta and the SMA was $30.82^{\circ} \pm 9.50^{\circ}$, the incidence of beak sign was $88.2 \%$, and the median diameter of the LRV at the AM portion and the mean diameter of the LRV at the hilar portion were 1.74 $\mathrm{mm}$ (range, $1.44-2.33 \mathrm{~mm}$ ) and $8.47 \pm 1.64 \mathrm{~mm}$, respectively. The median CR was 4.40 (range, 4.00-4.99) for NCS, and the median degree of compression was 0.79 (range, 0.77 $0.82)$ for NCS. There were significant differences between the two groups in the LRV MRI indices.

\section{Values of the LRV MRI indices in the diagnosis of NCS.}

The SMA angle, CR, and beak sign were plotted on an ROC curve (Figure 2). The results showed that the AUCs for SMA angle, CR, and beak sign in the diagnosis of NCS were $0.870,0.895$, and 0.878 , respectively. The best cutoff values for the SMA angle and CR were 36.8 and 3.99, 
Table 1 Baseline clinical characteristics of the study cohort

\begin{tabular}{lccc}
\hline Items & Nutcracker $(\mathrm{n}=58)$ & Controls $(\mathrm{n}=97)$ & $\mathrm{P}$ \\
\hline Female (\%) & $29(50.0)$ & $43(44.3)$ & 0.365 \\
Age, years & $11.0(10.0,13.0)$ & $11.0(10.0,13.0)$ & 0.651 \\
BMI $\left(\mathrm{kg} / \mathrm{m}^{2}\right)$ & $17.0 \pm 2.7$ & $18.1 \pm 3.0$ & 0.020 \\
Hematuria (\%) & $32(55.2)$ & $12(12.4)$ & $<0.001$ \\
Urinary protein (g/day) & $0.3(0.2,0.5)$ & $4(0.1,0.3)$ & 0.001 \\
Orthostatic proteinuria (\%) & $39(67.2)$ & $2(2.1)$ & $<0.001$ \\
Left flank pain (\%) & $9(15.5)$ & $5(5.2)$ & $<0.001$ \\
Abdominal pain (\%) & $11(19.0)$ & $<0.001$ & \\
\hline
\end{tabular}

BMI, body mass index.

Table 2 Imaging characteristics of left renal vein entrapment

\begin{tabular}{lccc}
\hline Items & Nutcracker $(\mathrm{n}=58)$ & Controls $(\mathrm{n}=97)$ & $\mathrm{P}$ \\
\hline SMA angle & $30.82 \pm 9.50$ & $59.59 \pm 26.42$ & $<0.001$ \\
Beak sign (\%) & $45(88.2)$ & $6(6.2)$ & $<0.001$ \\
Diameter at the hilar portion $(\mathrm{mm})$ & $8.47 \pm 1.64$ & $7.05 \pm 1.55$ & $<0.001$ \\
Diameter at the AM portion $(\mathrm{mm})$ & $1.74(1.44,2.33)$ & $2.17(1.97,2.91)$ & $<0.001$ \\
Compression ratio & $4.40(4.00,4.99)$ & $3.10(2.57,3.70)$ & $<0.001$ \\
Degree of compression & $0.79(0.77,0.82)$ & $0.67(0.60,0.72)$ & $<0.001<$ \\
\hline
\end{tabular}

SMA, superior mesenteric artery; AM, aortomesenteric.

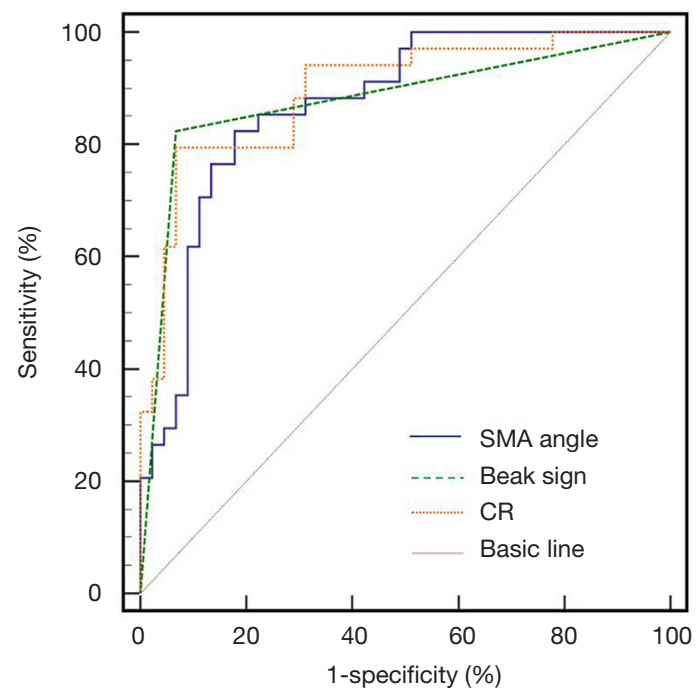

Figure 2 The ROC curve for the SMA angle, beak sign and CR. ROC, receiver operating characteristic; SMA, superior mesenteric artery; CR, compression ratio. respectively.

To evaluate the diagnostic value of the LRV MRI indices in the diagnosis of NCS, we performed further analysis based on the above results. Of the 155 patients, 65 patients $(41.9 \%)$ had SMA angle less than $36.8^{\circ}, 48$ patients with SMA angle $<36.8^{\circ}$ were diagnosed with NCS, and 10 patients with SMA angle $>36.8^{\circ}$ had NCS. Fiftyone patients $(32.9 \%)$ presented with beak sign, 45 patients with beak sign were diagnosed with NCS, and 13 patients without beak sign were diagnosed with NCS. Fifty-two patients $(33.5 \%)$ had $\mathrm{CR}>3.99,46$ patients with $\mathrm{CR}>3.99$ were diagnosed with NCS, and 12 patients without CR $>3.99$ were diagnosed with NCS. SMA angle $<36.8^{\circ}$ and beak sign were present at the same time in 41 patients (26.5\%), 39 patients with positive results were diagnosed with NCS and 19 patients with negative results were diagnosed with NCS. SMA angle $<36.8^{\circ}$ and CR $>3.99$ cooccurred in 39 patients $(25.2 \%), 35$ patients with positive results were diagnosed with NCS, and 23 patients with 

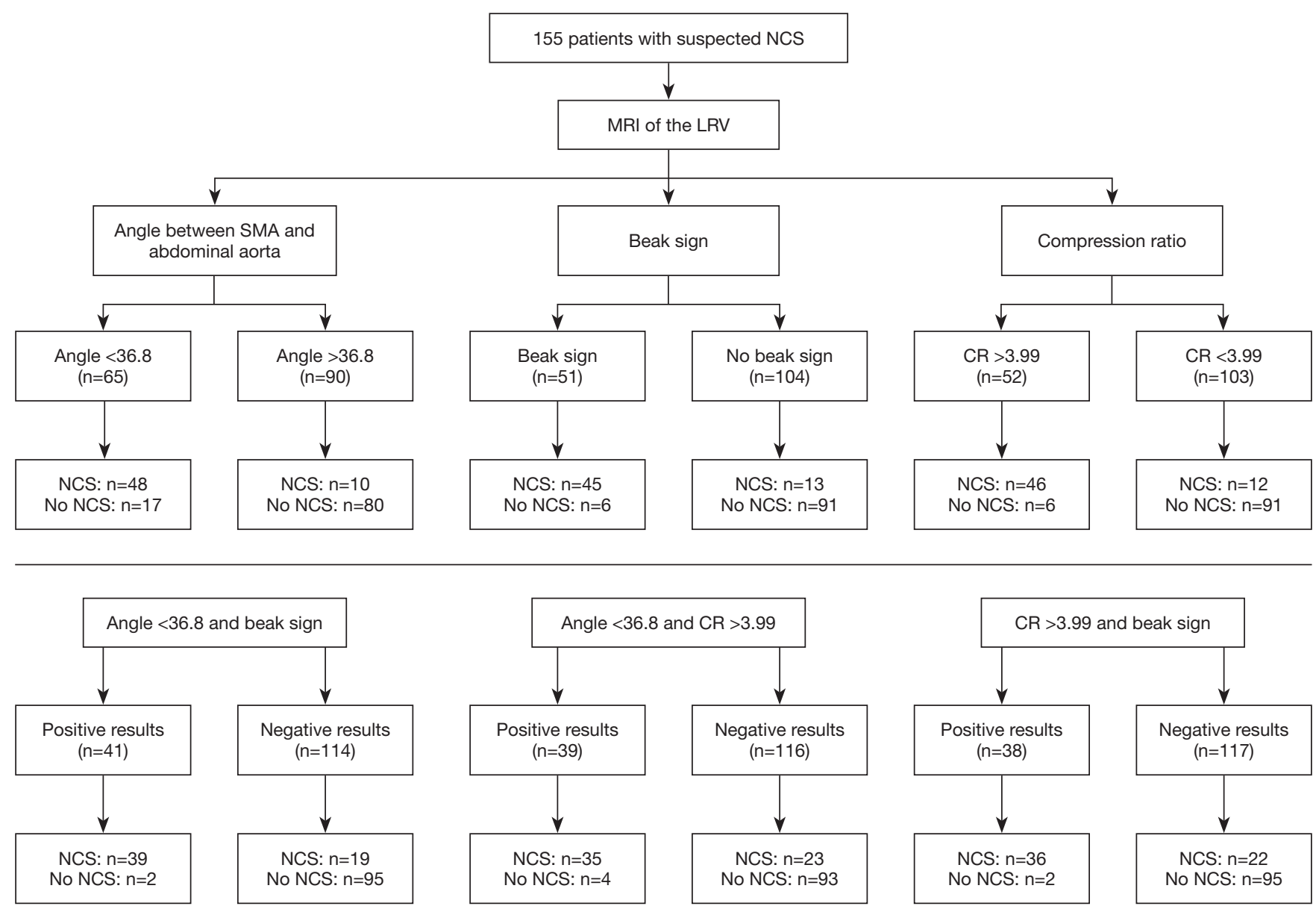

Figure 3 The distribution of the LRV MRI indices in patients. NCS, nutcracker syndrome; MRI, magnetic resonance imaging; LRV, left renal vein; SMA, superior mesenteric artery; CR, compression ratio.

negative results were diagnosed with NCS. Thirty-eight patients $(24.5 \%)$ had beak sign and CR $>3.99,36$ patients with positive results were diagnosed with NCS, and 22 patients with negative results were diagnosed with NCS (Figure 3).

Several indicators that were used to assess the diagnostic values of the MRI indices are listed in Table 3. The sensitivities of SMA angle $<36.8^{\circ}$, beak sign, CR $>3.99$, SMA angle $<36.8^{\circ}$ and beak sign, SMA angle $<36.8^{\circ}$ and CR $>3.99$, and beak sign and CR $>3.99$ were $82.8 \%$, $77.6 \%, 79.3 \%, 67.2 \%, 60.3 \%$ and $62.1 \%$, respectively. The specificities of SMA angle $<36.8^{\circ}$, beak sign, CR $>3.99$, SMA angle $<36.8^{\circ}$ and beak sign, SMA angle $<36.8^{\circ}$ and CR $>3.99$, and beak sign and CR $>3.99$ were $82.5 \%, 93.8 \%$, $93.8 \%, 97.9 \%, 95.9 \%$ and $97.9 \%$, respectively.

\section{Discussion}

NCS is a complex condition consisting of characteristic symptoms and signs that result from compression of the LRV. Limited data and lack of diagnostic consensus make the diagnosis of pediatric NCS difficult. This ambiguity in diagnosis is not favorable for patients with NCS who require endovascular stenting or renal venous replantation. Our results demonstrated that higher compression was related to OP, hematuria, left flank pain and abdominal pain (Table 1). However, the nutcracker anatomy is not always associated with the clinical signs and symptoms. Previous studies reported that children are more likely to present with asymptomatic NCS $(12,13)$. In this retrospective study, nine of 67 patients diagnosed with LRV compression did not meet the diagnostic criteria 
Table 3 The values of LRV MRI indices in the diagnosis of NCS

\begin{tabular}{|c|c|c|c|c|c|c|c|}
\hline Parameters & $\mathrm{Se}(95 \% \mathrm{Cl})$ & $\mathrm{Sp}(95 \% \mathrm{Cl})$ & PPV (95\% Cl) & NPV (95\% Cl) & PLR (95\% Cl) & NLR (95\% Cl) & YI (\%) \\
\hline Beak sign $^{b}$ & $77.6(0.7,0.9)$ & $93.8(0.9,1.0)$ & $88.2(0.8,1.00)$ & $87.5(0.8,1.0)$ & $12.5(10.6,58.8)$ & $0.2(0.1,0.2)$ & 71.4 \\
\hline $\mathrm{CR}>3.99^{\mathrm{c}}$ & $79.3(0.7,0.9)$ & $93.8(0.9,1.0)$ & $88.5(0.8,1.0)$ & $88.3(0.8,0.9)$ & $12.8(7.1,64.4)$ & $0.2(0.1,0.4)$ & 73.1 \\
\hline$a$ and $b$ & $67.2(0.6,0.8)$ & $97.9(0.9,1.0)$ & $95.1(0.9,1.0)$ & $83.3(0.8,0.9)$ & $32.0(13.9,83.3)$ & $0.3(0.2,0.4)$ & 65.1 \\
\hline $\mathrm{b}$ and $\mathrm{c}$ & $62.1(0.5,0.8)$ & $97.9(0.9,1.0)$ & $94.7(0.8,1.0)$ & $82.6(0.8,0.9)$ & $29.6(13.0,76.9)$ & $0.4(0.3,0.5)$ & 60.1 \\
\hline
\end{tabular}

LRV, left renal vein; MRI, magnetic resonance imaging; SMA, superior mesenteric artery; NCS, nutcracker syndrome; Se, sensitivity; CI, confidence interval; Sp, specificity; PPV, positive predictive value; NPV, negative predictive value; PLR, positive likelihood ratio; NLR, negative likelihood ratio; YI, Youden index; $\mathrm{CR}$, compression ratio. a: SMA angle <36.8; b: beak sign; c: CR >3.99.

for NCS. It is assumed that collateral connections can effectively drain the LRV and reduce the venous pressure, which may result in clinically asymptomatic patients. In addition, variations in the normal anatomy should be fully considered before the diagnosis of NCS. Compared to other imaging methods, MRI can provide visual images of the anatomy. It can demonstrate stenosis of the aorto-mesenteric region on the sagittal plane and LRV compression with pre-stenotic dilatation of the LRV (14). In addition, dorsolateral torsion of the left kidney, abnormal configuration of the SMA, abnormally high course of the LRV, and perirenal or gonadal vein varices can be imaged (2). Therefore, in children with clinically suspected NCS, MRI can be used as a preferred method to understand the anatomical structures in the aortomesenteric region. If asymptomatic NCS is present, the symptoms and signs of hematuria, proteinuria, abdominal pain, and left flank pain should be followed up.

In this study, the average age of onset was 11.0 (10.0, 13.0) years old, and this age is in the stage of rapid increase in body height and vertebral body maturation in adolescence, which reduces the aortomesenteric angle. Therefore, the anatomical structure of the aorto-mesenteric region in adults may be different than that in children. The mean aortomesenteric angles in children were $45.3^{\circ} \pm 21.6^{\circ}$ for girls and $45.8^{\circ} \pm 18.2^{\circ}$ for boys. The mean SMA-aorta distances in children were $11.5 \pm 4.5 \mathrm{~mm}$ for girls and $11.5 \pm 5.3 \mathrm{~mm}$ for boys (15). Most previous studies have focused on LRV compression in adults. A previous study in adults showed that the SMA angle needed to be less than $45^{\circ}$ to diagnose NCS and that an angle $<35^{\circ}$ was sufficient to make a definitive diagnosis (16). In another study in adults that focused on the diagnostic value of CT for NCS, an angle $<39^{\circ}$ had $92 \%$ sensitivity and $89 \%$ specificity for symptomatic NCS (17). Similarly, a cutoff of less than 36.8 had high sensitivity and specificity in our study, of $82.8 \%$ and $82.5 \%$, respectively. "Beak sign" is a sudden narrowing of the LRV at the aortomesenteric portion of a triangular shape, which may be the most useful parameter finding of MRI/CT in adults (2). In this study, we also found that "beak sign" is a highly accurate diagnostic indicator of NCS in children. Additionally, LRV stenosis is the most specific finding on axial imaging. A previous study in adults demonstrated that the CR was the most specific parameter in CT, with a CR $>4.9$ having the highest sensitivity $(66.7 \%)$ and specificity $(100.0 \%)$ for the diagnosis of NCS. Our results showed that a CR of $>3.99$ had a sensitivity of $79.3 \%$ and specificity of $93.8 \%$ for the diagnosis of NCS, which was lower than that in adults.

Our study also had limitations. Firstly, this is a singlecenter study, which is not representative enough. Secondly, due to the characteristics of hospitals and patients, the selection of research subjects could be biased, and the description of the population could be inaccurate. Thirdly, our study is a retrospective observational study. Due to the lack of clinical data of some patients, they could not be included in this study, so the sample size of this study was small. In addition, no long-term follow-up of the patients was conducted. Finally, the AUC was calculated only for the target population. External validation was not conducted.

\section{Conclusions}

This is the first study to explore the value of MRI indices of LRV entrapment in the diagnosis of NCS in children. Children with SMA angles less than 36.8, beak signs, and CRs greater than 3.99 should be highly suspected of having NCS. Among these parameters, "beak sign" showed the 
highest diagnostic accuracy by MRI, and the combination of any two of the above parameters achieved a higher specificity than the single parameters.

\section{Acknowledgments}

Thanks to Xiao Yang for polishing my article.

Funding: The authors acknowledge support from the Clinical Advanced Techniques, Primary Research \& Development Plan of Jiangsu Province (BE2017719), and the Pediatric Medical Innovation Team of Jiangsu Province (CXTDA2017022).

\section{Footnote}

Reporting Checklist: The authors have completed the STARD reporting checklist. Available at http://dx.doi.org/10.21037/ tp-20-466

Data Sharing Statement: Available at http://dx.doi. org/10.21037/tp-20-466

Peer Review File: Available at http://dx.doi.org/10.21037/tp20-466

Conflicts of Interest: All authors have completed the ICMJE uniform disclosure form (available at http://dx.doi. org/10.21037/tp-20-466). The authors have no conflicts of interest to declare.

Ethical Statement: The authors are accountable for all aspects of the work in ensuring that questions related to the accuracy or integrity of any part of the work are appropriately investigated and resolved. The study was approved by the Institutional Ethics Committee of Nanjing Medical University (2020NZGKJ-004) and individual consent for this retrospective analysis was waived. All data were obtained via electronic medical records and a database review and were de-identified (the patient's name was replaced with an identification code, and the patient's private information was deleted before the analysis) to protect patient privacy. The study was conducted in accordance with the Declaration of Helsinki (as revised in 2013).

Open Access Statement: This is an Open Access article distributed in accordance with the Creative Commons Attribution-NonCommercial-NoDerivs 4.0 International
License (CC BY-NC-ND 4.0), which permits the noncommercial replication and distribution of the article with the strict proviso that no changes or edits are made and the original work is properly cited (including links to both the formal publication through the relevant DOI and the license). See: https://creativecommons.org/licenses/by-nc-nd/4.0/.

\section{References}

1. Ananthan K, Onida S, Davies AH. Nutcracker Syndrome: An Update on Current Diagnostic Criteria and Management Guidelines. Eur J Vasc Endovasc Surg 2017;53:886-94.

2. Shin JI, Lee JS, Kim MJ. The prevalence, physical characteristics and diagnosis of nutcracker syndrome. Eur J Vasc Endovasc Surg 2006;32:335-6.

3. Chen YM, Wang IK, Ng KK, et al. Nutcracker syndrome: an overlooked cause of hematuria. Chang Gung Med J 2002;25:700-5.

4. Mahmood SK, Oliveira GR, Rosovsky RP. An easily missed diagnosis: flank pain and nutcracker syndrome. BMJ Case Rep 2013;2013:bcr2013009447.

5. Shin JI, Park JM, Lee JS, et al. Effect of renal Doppler ultrasound on the detection of nutcracker syndrome in children with hematuria. Eur J Pediatr 2007;166:399-404.

6. He Y, Wu Z, Chen S, et al. Nutcracker syndrome--how well do we know it? Urology 2014;83:12-7.

7. Ananthan K. Response to "Re. Nutcracker Syndrome: An Update on Current Diagnostic Criteria and Management Guidelines". Eur J Vasc Endovasc Surg 2018;55:741-2.

8. Yun SJ, Lee JM, Nam DH, et al. Discriminating renal nutcracker syndrome from asymptomatic nutcracker phenomenon using multidetector computed tomography. Abdom Radiol (NY) 2016;41:1580-8.

9. Arslan Z, Koyun M, Erengin H, et al. Orthostatic proteinuria: an overestimated phenomenon? Pediatr Nephrol 2020;35:1935-40.

10. Niu XL, Wu Y, Hao S, et al. Value of micro-proteinuria in combination with ultrasonography of the left renal vein in the diagnosis of orthostatic proteinuria. Ann Transl Med 2019;7:780.

11. Hangge PT, Gupta N, Khurana A, et al. Degree of Left Renal Vein Compression Predicts Nutcracker Syndrome. J Clin Med 2018;7:107.

12. Penfold D, Lotfollahzadeh S. Nutcracker Syndrome. In: StatPearls [Internet]. Treasure Island (FL): StatPearls Publishing, 2021.

13. Nalcacioglu H, Ceyhan BM, Tekcan D, et al. Nutcracker 
Syndrome in Children: Role of Doppler Ultrasonographic Indices in Detecting the Pattern of Symptoms. J Clin Med 2018;7:214.

14. Kurklinsky AK, Rooke TW. Nutcracker phenomenon and nutcracker syndrome. Mayo Clin Proc 2010;85:552-9.

15. Arthurs OJ, Mehta U, Set PA. Nutcracker and SMA syndromes: What is the normal SMA angle in children? Eur J Radiol 2012;81:e854-61.

Cite this article as: Wang R, Wang M, Xia Z, Gao C, Kuang Q, Fang X, Yu M, Peng Y, Li X, Wei Y, Ju T. Value of magnetic resonance imaging indices of left renal vein entrapment in the diagnosis of nutcracker syndrome in children. Transl Pediatr 2021;10(5):1285-1293. doi: 10.21037/tp-20-466
16. Polguj M, Topol M, Majos A. An unusual case of left venous renal entrapment syndrome: a new type of nutcracker phenomenon? Surg Radiol Anat 2013;35:263-7.

17. Kim KW, Cho JY, Kim SH, et al. Diagnostic value of computed tomographic findings of nutcracker syndrome: correlation with renal venography and renocaval pressure gradients. Eur J Radiol 2011;80:648-54. 